\title{
Arterial Baroreflex Buffering of Sympathetic Activation during Exercise-induced Elevations in Arterial Pressure
}

\author{
Urs Scherrer, ${ }^{\star}$ Susan L. Pryor, ${ }^{\star}$ Loren A. Bertocci, ${ }^{\star}$ and Ronald G. Victor ${ }^{\star}$ \\ ${ }^{*}$ Department of Internal Medicine (Cardiology Division), ${ }^{*}$ Harry S. Moss Heart Center, and the ${ }^{\ddagger}$ Biomedical Nuclear Magnetic \\ Resonance Center, University of Texas Southwestern Medical Center, Dallas, Texas 75235
}

\begin{abstract}
Static muscle contraction activates metabolically sensitive muscle afferents that reflexively increase sympathetic nerve activity and arterial pressure. To determine if this contractioninduced reflex is modulated by the sinoaortic baroreflex, we performed microelectrode recordings of sympathetic nerve activity to resting leg muscle during static handgrip in humans while attempting to clamp the level of baroreflex stimulation by controlling the exercise-induced rise in blood pressure with pharmacologic agents. The principal new finding is that partial pharmacologic suppression of the rise in blood pressure during static handgrip (nitroprusside infusion) augmented the exercise-induced increases in heart rate and sympathetic activity by $>\mathbf{3 0 0} \%$. Pharmacologic accentuation of the exercise-induced rise in blood pressure (phenylephrine infusion) attenuated these reflex increases by $>\mathbf{5 0 \%}$. In contrast, these pharmacologic manipulations in arterial pressure had little or no effect on: (a) forearm muscle cell $\mathrm{pH}$, an index of the metabolic stimulus to skeletal muscle afferents; or (b) central venous pressure, an index of the mechanical stimulus to cardiopulmonary afferents.

We conclude that in humans the sinoaortic baroreflex is much more effective than previously thought in buffering the reflex sympathetic activation caused by static muscle contraction. (J. Clin. Invest. 1990. 86:1855-1861.) Key words: microneurography $\bullet$ sympathetic nerve activity $\bullet$ baroreceptor reflexes • static exercise
\end{abstract}

\section{Introduction}

Static exercise evokes large increases in arterial pressure that are caused by decreases in parasympathetic and increases in sympathetic efferent activity $(1,2)$. There is abundant evidence that these autonomic adjustments are triggered both by the central neural drive associated with voluntary motor effort, "central command" $(3,4)$, and by a peripheral reflex caused by stimulation of mechanically and chemically sensitive afferents

A preliminary report of this work was presented at the 1989 meeting of the Federation of American Societies for Experimental Biology, New Orleans, LA, April 1989, and published in 1989 in abstract form (FASEB [Fed. Am. Soc. Exp. Biol.] J. 3:A248).

Address reprint requests to Dr. R. Victor, Cardiology Division, U.T. Southwestern Medical Center, 5323 Harry Hines Blvd., Dallas, TX 75235-9034. 1990.

Received for publication 20 March 1990 and in revised form 5 July

J. Clin. Invest.

(C) The American Society for Clinical Investigation, Inc.

0021-9738/90/12/1855/07 \$2.00

Volume 86, December 1990, 1855-1861 in contracting skeletal muscle (5-7). However, there is little consensus regarding the degree to which these excitatory neural inputs are modulated by the inhibitory influence of the baroreceptors (8-17).

Accordingly, the goal of this study was to test the hypothesis that sinoaortic baroreceptors, when stimulated by the large increases in arterial pressure that accompany static exercise, buffer the exercise-induced increases in heart rate and sympathetic nerve activity. To test this hypothesis, we performed microelectrode recordings of sympathetic nerve discharge to resting skeletal muscle in conscious, exercising humans while attempting to clamp the level of sinoarotic baroreceptor stimulation by controlling the exercise-induced rise in blood pressure with pharmacologic agents.

\section{Methods}

\section{Subjects}

Eight healthy male volunteers, ages $23-33$ yr, participated in this study after providing informed written consent. All subjects were normotensive (supine blood pressures $<140 / 90 \mathrm{mmHg}$ ), were taking no medications, and had no evidence of cardiopulmonary disease by history and physical examination at the time of the study. The experimental protocol was approved by the Institutional Review Board for human investigation.

\section{General procedures}

Subjects were studied in the supine position. Heart rate (electrocardiogram), respiratory excursions (pneumograph), force of muscle contraction (Stoelting handgrip dynamometer; Stoelting Co., Chicago, IL), and efferent muscle sympathetic nerve activity (MSNA), ${ }^{1}$ were recorded continuously on a Gould ES 1,000 electrostatic recorder (Gould Inc., Oxnard, CA) and on a TEAC R 71 tape recorder (TEAC Corp., Tokyo, Japan). Respiratory excursions were monitored to detect inadvertent performance of a Valsalva maneuver or prolonged expiration; these respiratory maneuvers can markedly stimulate muscle sympathetic outflow (18). In all experiments blood pressure was measured over the popliteal artery in the left leg with an automated sphygmomanometric system (Dinamap oscillometric sphygmomanometer; Critikon Inc., Tampa, FL) that recorded blood pressure once every $30 \mathrm{~s}$. In one experiment, arterial pressure was recorded continuously with an indwelling catheter in the radial artery. Mean arterial pressure was calculated as diastolic pressure plus $1 / 3$ pulse pressure. In some experiments, central venous pressure was measured with a catheter advanced from an antecubital vein into an intrathoracic vein.

\section{Recording of sympathetic nerve activity}

Multiunit recordings of postganglionic sympathetic nerve activity were obtained with unipolar tungsten microelectrodes inserted selectively into muscle nerve fascicles of the peroneal nerve posterior to the fibular head by the microneurographic technique of Vallbo, Hagbarth, et

1. Abbreviations used in this paper: MSNA, muscle sympathetic nerve activity; MVC, maximal voluntary contraction; NMR, nuclear magnetic resonance; $\mathrm{PCr}$, phosphocreatine; $\mathrm{Pi}$, inorganic phosphate. 
al. (19). The neural signals were amplified (by 20-50 $\times 10^{3}$ ), filtered (bandwidth 700-2,000 Hz), rectified, and integrated (time constant of $0.1 \mathrm{~s})$ to obtain a mean voltage display of sympathetic activity. A recording of MSNA was considered acceptable when the neurograms revealed spontaneous, pulse-synchronous bursts of neural activity that increased during the Valsalva maneuver, but not during arousal stimuli (loud noise, skin pinch). Sympathetic bursts were identified by inspection of the filtered and mean voltage neurograms: the interobserver and intraobserver variability in identifying bursts is $<10 \%$ and $<5 \%$, respectively (20). Nerve traffic was expressed both as bursts per minute, an index of the frequency of activity, and as bursts per minute times mean burst amplitude, an index of integrated (total) activity.

\section{${ }^{31} P$ NMR spectroscopy}

Intracellular $\mathrm{pH}$ and high energy phosphates were monitored in the exercising forearm muscles using phosphorus nuclear magnetic resonance spectroscopy ( ${ }^{31} \mathrm{P}$ NMR). With the subject supine, the exercising arm was placed in a $30-\mathrm{cm}$ horizontal bore 1.9 Tesla superconducting magnet (Oxford Instruments, Oxford, UK) interfaced to an NT-80 console (GE/NMR, Fremont, CA). A 2.0-cm surface coil, tuned to the ${ }^{31} \mathrm{P}$ resonance frequency of $32.54 \mathrm{MHz}$, was placed over the flexor digitorum profundus muscle. Magnetic resonance spectra were acquired in 30-s intervals and represented the time average of 20 acquisitions. Intracellular $\mathrm{pH}$ was estimated from the chemical shift of inorganic phosphate $(\mathrm{Pi})$ relative to phosphocreatine $(\mathrm{PCr})(21)$. The contraction-induced fall in forearm muscle cell $\mathrm{pH}$ was used as an index of muscle hypoperfusion $(22,23)$ and as an index of muscle metaboreceptor reflex stimulation $(24,25)$.

\section{Static handgrip and posthandgrip forearm circulatory arrest}

At the beginning of each experiment, the subject's maximal voluntary contraction (MVC) was determined using a handgrip dynamometer. Subjects performed static handgrip at 33\% MVC for 2 min, aided by visual feedback of force output. During the last $5 \mathrm{~s}$ of static handgrip, a pneumatic cuff was inflated to suprasystolic pressure $(250 \mathrm{mmHg})$ on the exercising upper arm to arrest the forearm circulation. The arrested circulation was maintained for an additional $2 \mathrm{~min}$ in the postexercise period. This maneuver was used to maintain the metabolic stimulation of muscle afferents while eliminating central command with muscular relaxation (20).

\section{Intravenous infusions of vasoactive drugs}

Nitroprusside (in a dose of $1.0-3.0 \mu \mathrm{g} / \mathrm{kg}$ per minute) or phenylephrine (in a dose of $1.0-2.5 \mu \mathrm{g} / \mathrm{min}$ per kilogram) were infused into a forearm vein using an infusion pump (Harvard Apparatus Co., Inc., The Ealing Corp., South Natick, MA). Nitroprusside or phenylephrine were infused at progressively faster rates during 2 min of sustained handgrip in order to attenuate or augment the rise in blood pressure during the exercise. Infusions were continued during posthandgrip forearm circulatory arrest in order to maintain a constant level of blood pressure.

\section{Experimental protocols}

Protocol 1: Effects of pharmacologic manipulations of blood pressure on MSNA and heart rate responses to handgrip. In all eight subjects, we recorded MSNA from the right peroneal nerve during 2 min of static handgrip at 33\% MVC followed by 2 min of forearm circulatory arrest performed alone, and during concomitant intravenous infusion of either nitroprusside or phenylephrine. In each subject, the infusion rates of the two drugs were titrated individually to attenuate or augment the normal handgrip-induced rise in blood pressure by about $50 \%$, i.e., by $\sim 10-15 \mathrm{mmHg}$ in either direction. Infusions were started immediately after handgrip was initiated. During infusion of nitroprusside, particular care was taken not to lower blood pressure below the resting baseline level. Subjects rested quietly for $15 \mathrm{~min}$ before starting the experimental interventions. There were at least $30 \mathrm{~min}$ of rest between each experimental protocol.

To determine effects of these interventions on the latency in onset of the MSNA response during $2 \mathrm{~min}$ of static handgrip, in each subject total MSNA was measured in 12 consecutive 10-s intervals; onset latency was measured as the time (rounded to tens of seconds) between the onset of handgrip to the onset of a $50 \%$ increase in MSNA over control.

Protocol 2: Comparative effects of nitroprusside-induced reductions in blood pressure on MSNA at rest and during handgrip. In five of the eight subjects, we measured MSNA during infusion of nitroprusside under resting conditions (i.e., without handgrip). In these experiments, the dose of nitroprusside was titrated to lower the resting level of blood pressure by approximately $10-15 \mathrm{mmHg}$, i.e., by the same amount as that achieved when nitroprusside was infused during handgrip. The aim of this protocol was to determine if the augmentation in handgrip-induced sympathetic activation caused by reduction in baroreceptor stimulation was greater than the simple algebraic sum of the increases in sympathetic activity caused by handgrip alone and that caused by reduction in baroreceptor stimulation alone.

Protocol 3: Effects of pharmacologic manipulations of the exerciseinduced rise in blood pressure on intracellular $\mathrm{pH}$ in contracting forearm muscle. The aim of this protocol was to determine if drug-induced manipulations in the level of blood pressure during static handgrip altered forearm muscle cell $\mathrm{pH}$, an index of the metabolic stimulus to skeletal muscle afferents. In five of the eight subjects, we repeated the static handgrip, both alone and in combination with infusion of nitroprusside and phenylephrine, while we used ${ }^{31} \mathrm{P}$ NMR to monitor intracellular $\mathrm{pH}$ and high energy phosphates in the exercising forearm muscles.

Protocol 4: Effects of nitroprusside infusion on central venous pressure during handgrip. To determine if infusion of nitroprusside during handgrip decreased central venous pressure, an index of the mechanical stimulus to cardiopulmonary afferents, we performed direct measurements of central venous pressure in three of the eight subjects. To offset any such reduction in central venous pressure caused by nitroprusside, we performed additional experiments in which the nitroprusside infusion was accompanied by rapid intravenous infusion of approximately $500 \mathrm{ml}$ of normal saline. The effects on MSNA responses to handgrip of nitroprusside infusion alone were compared with effects of concomitant infusion of nitroprusside and saline.

\section{Data analysis}

Statistical analysis was performed using repeated measures of analysis of variance with the Bonferroni adjustment for multiple comparisons. A $P$ value $<0.05$ was considered statistically significant. Results are expressed as mean $\pm \mathrm{SE}$.

\section{Results}

2 min of static handgrip (33\% MVC) alone increased mean arterial pressure by $24 \pm 4 \mathrm{mmHg}$, MSNA by $233 \pm 49 \%$, and heart rate by $14 \pm 2$ beats per minute. Blunting the normal rise in arterial pressure during handgrip by $50 \%$ with infusion of nitroprusside caused a fourfold augmentation in the MSNA response and a threefold augmentation in the heart rate response to this level of handgrip (Table I and Fig. 1). Accentuating the normal rise in arterial pressure by $50 \%$ with infusion of phenylephrine caused a $60-70 \%$ attenuation of the handgrip-induced increases in MSNA and heart rate (Table I and Fig. 1). The latency in onset of the MSNA response to static handgrip was shortened from $56 \pm 5$ to $35 \pm 4 \mathrm{~s}$ with concomitant infusion of nitroprusside $(P<0.05)$ and lengthened to $95 \pm 12 \mathrm{~s}$ with infusion of phenylephrine $(P<0.05)$.

The increases in arterial pressure and MSNA observed during handgrip were maintained, with minimal attenuation, during posthandgrip forearm circulatory arrest performed alone or in combination with infusion of nitroprusside or phenylephrine (Table I and Fig. 2). In contrast, handgrip-in- 
Table I. Responses to Static Handgrip (33\% Maximal Voluntary Contraction) and Posthandgrip Forearm Circulatory Arrest Performed Alone and During Infusion of Nitroprusside or Phenylephrine

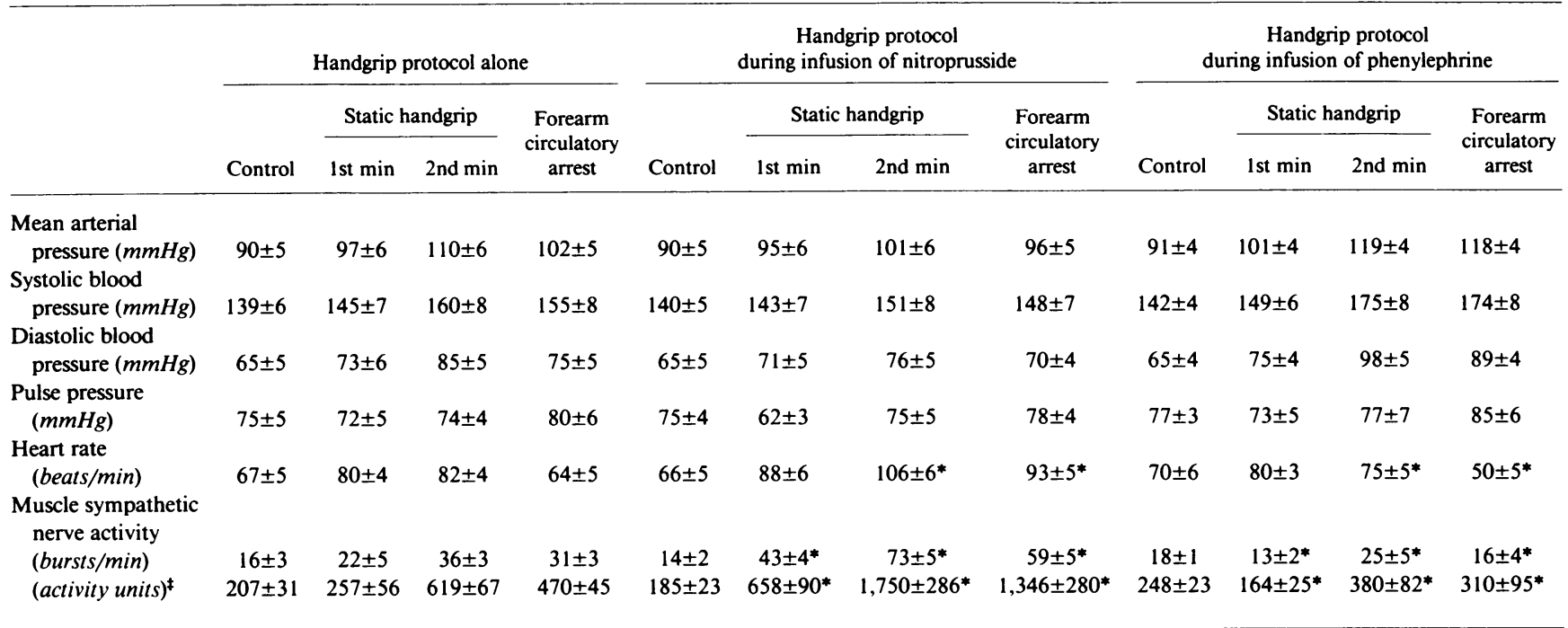

Entries are mean $\pm \mathrm{SE}$ for eight subjects. Measurements represent the average value of $2 \mathrm{~min}$ of control; peak responses during the last $30 \mathrm{~s}$ of each minute of handgrip; and the average of $2 \mathrm{~min}$ of forearm circulatory arrest. ${ }^{*} P<0.05 \mathrm{vs}$. handgrip alone. ${ }^{\ddagger}$ Muscle sympathetic nerve activity given in units (bursts/min $\cdot$ mean burst amplitude).
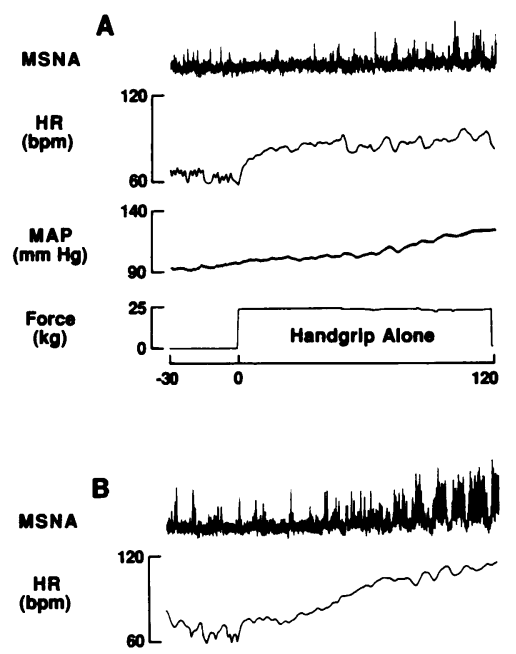

$\underset{\operatorname{maP}}{\operatorname{maP}}$

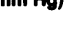

Force
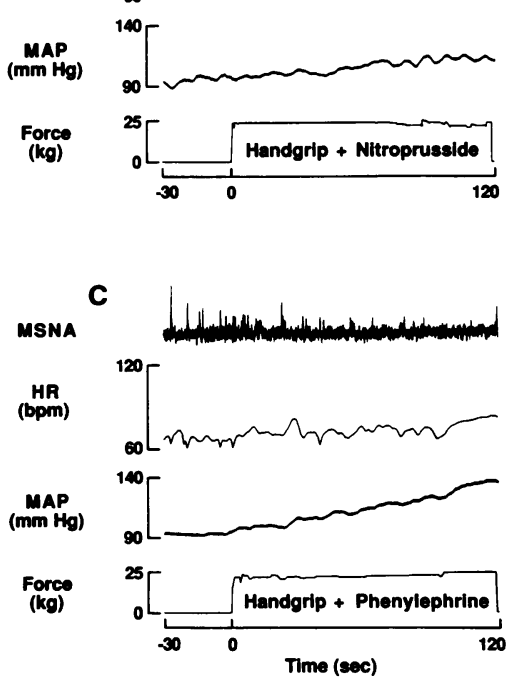

Figure 1. Segments of an illustrative record from one subject showing effects of pharmacologic manipulations in arterial pressure on MSNA responses to static handgrip at $33 \%$ MVC. Panels show the mean voltage neurogram of MSNA, heart rate $(H R)$, mean arterial pressure (MAP); and the force tracing when handgrip was performed alone $(A)$, during infusion of nitroprusside $(B)$, and during infusion of phenylephrine $(C)$. For purposes of illustration, the record has been transcribed from FM tape at a paper speed of 1 $\mathrm{mm} / \mathrm{s}$ to demonstrate the entire handgrip sequence. Modest alterations in the level of blood pressure during handgrip had profound effects on the exerciseinduced increases in both heart rate and muscle sympathetic outflow. duced increases in heart rate: $(a)$ normally returned to baseline ( $64 \pm 5$ beats per minute) during posthandgrip forearm circulatory arrest; but $(b)$ were partially maintained $(93 \pm 5$ beats per minute) during forearm circulatory arrest performed with infusion of nitroprusside; and $(c)$ were replaced by bradycardia ( $50 \pm 5$ beats per minute) during forearm circulatory arrest with infusion of phenylephrine.

Fig. 3 compares effects of nitroprusside-induced reduction in blood pressure on increases in MSNA at rest and during handgrip. For a parallel reduction in mean arterial pressure of $12 \mathrm{mmHg}$, nitroprusside caused a sixfold greater increase in MSNA when the drug was infused during static handgrip than when infused at rest. The increases in MSNA caused by handgrip performed during infusion of nitroprusside, $876 \pm 116 \%$, were more than two times greater $(P<0.05)$ than the simple algebraic sum of the increases caused by handgrip alone, $265 \pm 56 \%$, and those caused by nitroprusside infusion alone, $116 \pm 16 \%$.

Alterations in the rise in arterial pressure during handgrip with infusions of nitroprusside or phenylephrine had no detectable effects on the contraction-induced fall in forearm muscle cell pH (Table II and Fig. 4).

During static handgrip, central venous pressure (measured in three subjects) decreased from $5.0 \pm 0.3$ to $3.8 \pm 1.0 \mathrm{mmHg}$ during infusion of nitroprusside (Fig. 5). Offsetting this small decrease in central venous pressure with an intravenous infusion of saline had no effect on the augmentation of the MSNA response to handgrip produced by the nitroprusside.

\section{Discussion}

The role played by the sinoaortic baroreflex in the regulation of sympathetic neural outflow during exercise previously has been difficult to elucidate using indirect indices of sympathetic 
a

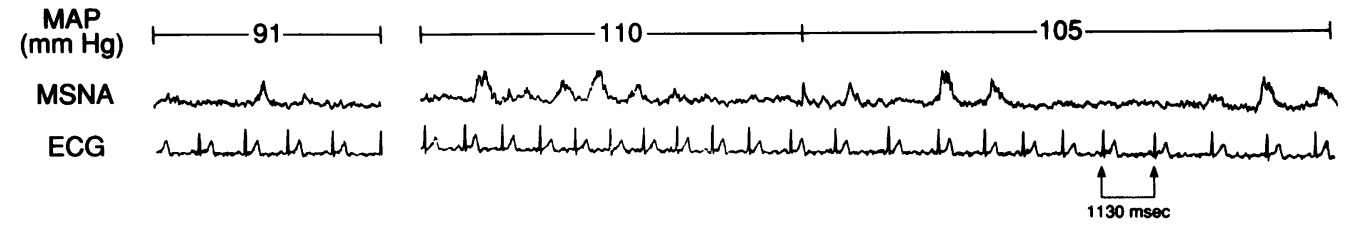

b

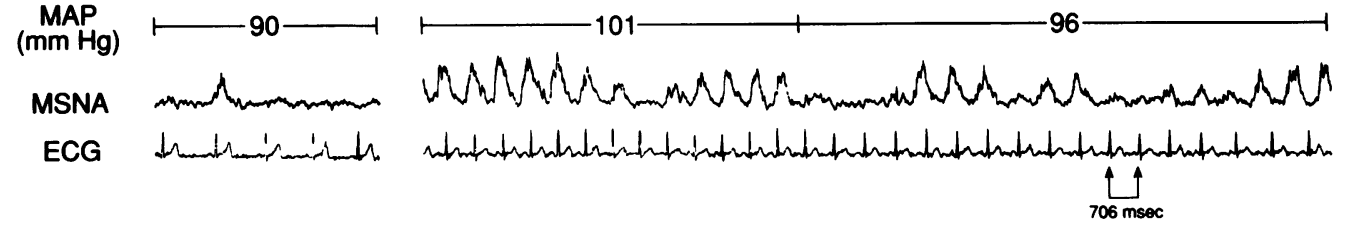

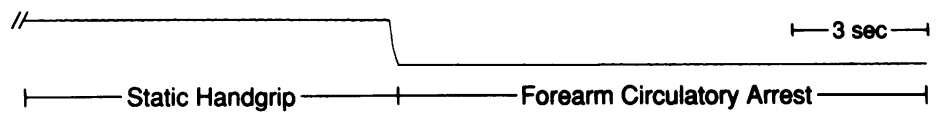

Figure 2. Segments of an original record in one subject showing effects of nitroprusside infusion on MSNA and heart rate during the last $8 \mathrm{~s}$ of static handgrip followed by arrest of the forearm circulation. The figure shows values of mean arterial pressure $(M A P)$, the mean voltage neurogram of MSNA, and the corresponding electrocardiogram $(E C G)$. The numbers shown in brackets below the ECG represent the $\mathbf{R}-\mathbf{R}$ intervals in milliseconds ( $m s e c$ ). Handgrip was followed by fore-

arm circulatory arrest $(a)$ under normal conditions and $(b)$ during concomitant infusion of nitroprusside to attenuate the handgrip-induced rise in arterial pressure by $50 \%$. Under normal conditions (a), handgrip-induced increases in MSNA were maintained during posthandgrip forearm circulatory arrest whereas heart rate returned rapidly to control. During infusion of nitroprusside $(b)$, handgrip evoked augmented increases in MSNA and heart rate that were both partially maintained during posthandgrip forearm circulatory arrest.

activity (8-16). The ability to measure sympathetic nerve discharge directly in humans provided the opportunity to develop a straightforward experimental approach to study this problem. The major new finding is that during static handgrip partial pharmacologic suppression of the exercise-induced rise in blood pressure augmented the reflex increases in muscle sympathetic outflow and heart rate by more than $300 \%$. Conversely, moderate accentuation of the exercise-induced rise in blood pressure attenuated these reflex increases by more than
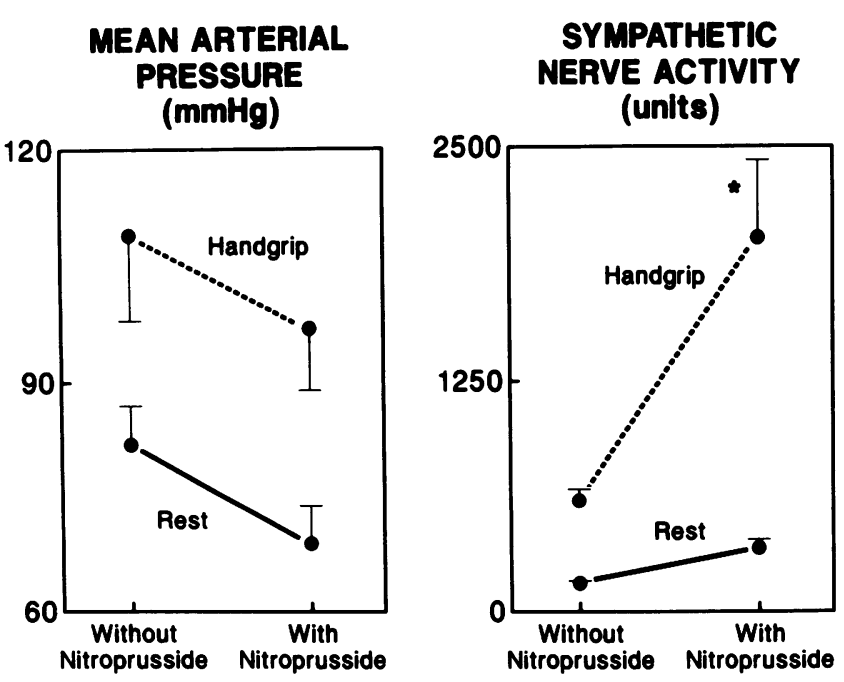

Figure 3. Comparative effects of nitroprusside-induced reductions in mean arterial pressure on increases in sympathetic nerve activity at rest and during handgrip. For a parallel reduction in arterial pressure, nitroprusside caused a sixfold greater augmentation in MSNA when the drug was infused during handgrip than when infused at rest. $\left({ }^{*}\right)$ Indicates that the increases in MSNA caused by handgrip performed during infusion of nitroprusside were significantly $(P$ $<0.05$ ) greater than the simple algebraic sum of the increases caused by handgrip alone, and by infusion of nitroprusside alone. Entries are mean $\pm S E$ for five subjects.
$50 \%$. The findings provide the first evidence in humans that the sinoaortic baroreflex, when stimulated by the large rise in mean arterial pressure that normally accompanies static exercise, markedly limits the increases in muscle sympathetic outflow, as well as heart rate, evoked by this form of exercise.

This interpretation is predicated on the assumption that the reflex autonomic effects of our pharmacologic interventions indeed were caused by sinoaortic baroreceptors. We therefore examined the alternative possibility that the pharmacologic manipulations in systemic arterial pressure also might have altered the stimulus either to the excitatory reflex caused by skeletal muscle afferents or to the inhibitory reflex caused by cardiopulmonary afferents.

During static contraction in anesthetized animals, metaboreceptor muscle afferents are activated by ischemic metabolites such as hydrogen ions that accumulate in the muscle interstitium when blood flow fails to meet the metabolic demands of the contracting muscle $(26,27)$. During static handgrip in conscious humans, stimulation of these afferents is thought to be the primary mechanism that causes sympathetic activation in resting skeletal muscle $(20,28)$, a response that is closely coupled to the cellular accumulation of hydrogen ions in contracting forearm muscle $(24,25)$. The increases in arterial pressure, which result in part from this reflex sympathetic activation, are thought to partially correct the hypoperfusion (and acidosis) in statically contracted muscle and thus minimize additional stimulation of the afferents (27). Thus, in our experiments, attenuation of the normal exercise-induced rise in arterial pressure (infusion of nitroprusside) might have augmented this metabolically-generated muscle afferent reflex by exacerbating muscle hypoperfusion. This possibility, however, is unlikely because infusion of nitroprusside, or of phenylephrine, had no significant effect on the contraction-induced fall in forearm muscle cell $\mathrm{pH}$.

Another possibility is that our interventions altered the stimulation of inhibitory cardiopulmonary mechanoreceptor afferents. During static exercise, increases in central venous pressure should stimulate these afferents; however, concomi- 


\begin{tabular}{|c|c|c|c|c|c|c|}
\hline & \multicolumn{2}{|c|}{ Handgrip alone } & \multicolumn{2}{|c|}{$\begin{array}{l}\text { Handgrip during infusion } \\
\text { of nitroprusside }\end{array}$} & \multicolumn{2}{|c|}{$\begin{array}{l}\text { Handgrip during infusion } \\
\text { of phenylephrine }\end{array}$} \\
\hline & Control & Handgrip & Control & Handgrip & Control & Handgrip \\
\hline Mean arterial pressure $(\mathrm{mmHg})$ & $94 \pm 4$ & $118 \pm 3$ & $95 \pm 5$ & $108 \pm 4$ & $95 \pm 3$ & $127 \pm 2$ \\
\hline $\mathrm{H}+($ nmol $)$ & $79 \pm 2$ & $161 \pm 9$ & $79 \pm 4$ & $146 \pm 19$ & $70 \pm 5$ & $209 \pm 24$ \\
\hline $\mathrm{pH}$ & $7.1 \pm 0.1$ & $6.8 \pm 0.1$ & $7.1 \pm 0.1$ & $6.8 \pm 0.1$ & $7.2 \pm 0.1$ & $6.7 \pm 0.1$ \\
\hline
\end{tabular}

Entries are mean \pm SE for five subjects. Measurements represent the average value of $2 \mathrm{~min}$ of control; and peak responses during the last $30 \mathrm{~s}$ of the second minute of handgrip exercise.
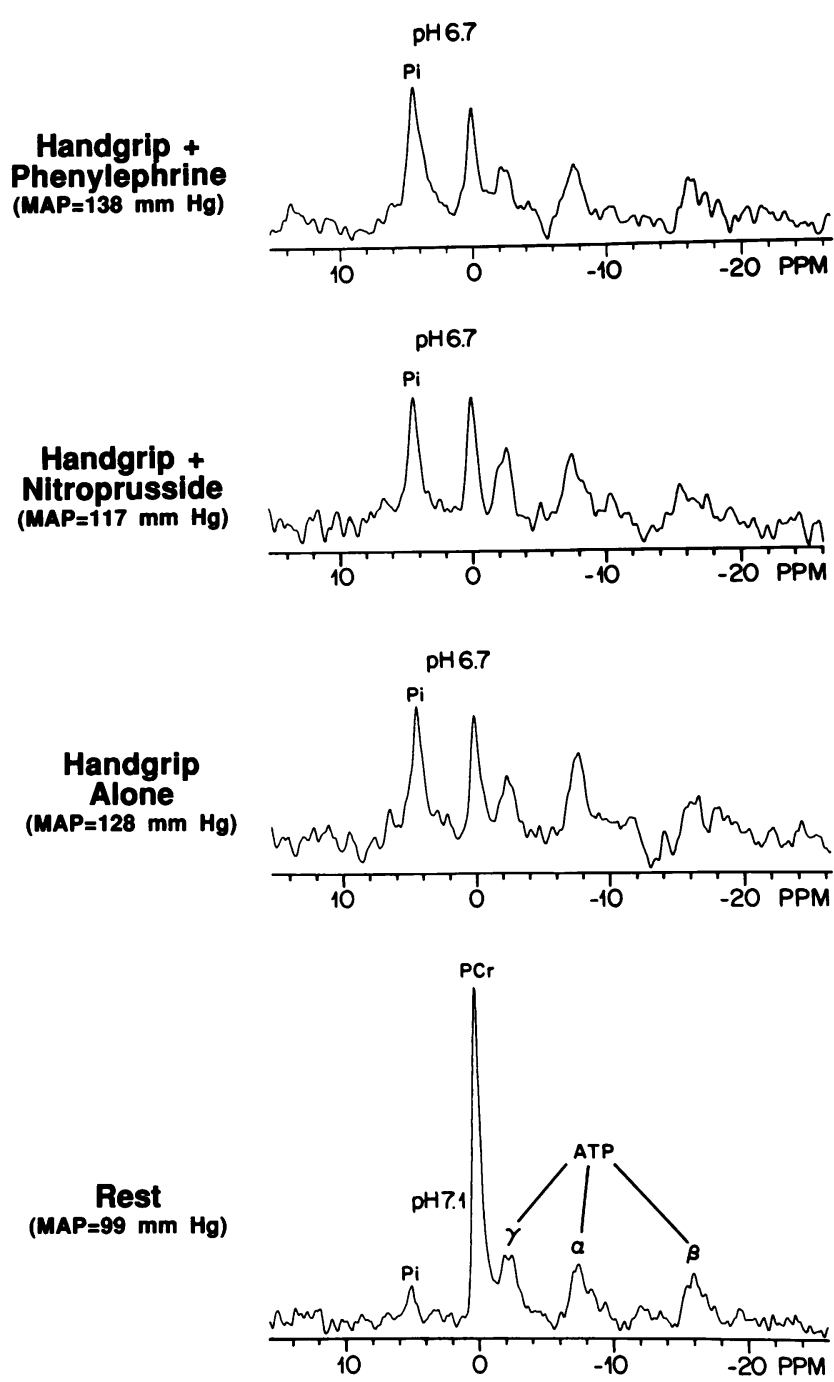

Figure 4. Original ${ }^{31} \mathrm{P}$ NMR spectra and corresponding values for mean arterial pressure $(M A P)$ in one subject at rest and during the last $30 \mathrm{~s}$ of $2 \mathrm{~min}$ of static handgrip at $33 \%$ of maximum performed alone and during infusion of either nitroprusside or phenylephrine. On the NMR spectra, signal intensities are proportional to the intracellular concentrations of inorganic phosphate $(P i)$, phosphocreatine $(P C r)$ and adenosine triphosphate $(A T P) ; \mathrm{pH}$ is calculated from the chemical shift of $\mathrm{Pi}$ relative to $\mathrm{PCr}$. Augmenting or attenuating the normal rise in blood pressure during handgrip by about $10 \mathrm{mmHg}$ had no detectable effect on either the fall in muscle cell $\mathrm{pH}$ or the utilization of high energy phosphates in the contracting forearm muscles of this subject. tant infusion of nitroprusside, a mixed venous-arterial vasodilator, might have offset the exercise-induced increase in central venous pressure. Thus, augmentation of the MSNA response to static handgrip during infusion of nitroprusside could have been caused by a relative unloading of cardiopulmonary, as well as of sinoaortic, afferents. This possibility is unlikely because three recent studies have shown that, without decreases in arterial pressure, decreases in central venous pressure alone do not augment the MSNA response to static handgrip (29-31). This interpretation is strengthened by our experiments performed on three subjects in whom the ability of nitroprusside to potentiate MSNA responses to handgrip was preserved when the small nitroprusside-induced fall in central venous pressure was prevented with an intravenous infusion of saline.

Our data therefore strongly suggest that sinoaortic baroreflex stimulation is a major determinant of sympathetic discharge during static exercise. This conclusion differs from that of a previous study in which stimulation of carotid baroreceptors with external application of negative pressure had no de-
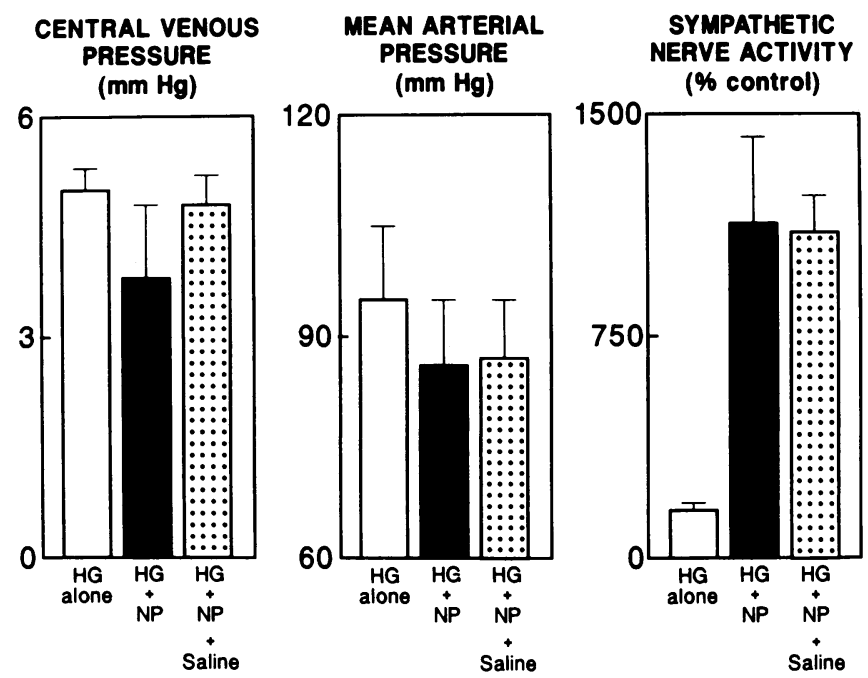

Figure 5. Comparison of sympathetic nerve responses to handgrip $(H G)$ performed alone or during infusion of nitroprusside $(N P)$ with or without concomitant infusion of saline. Central venous pressure tended to decrease slightly during infusion of nitroprusside. Offsetting this small decrease in central venous pressure with an intravenous infusion of saline had no effect on the augmentation of the sympathetic response to handgrip produced by nitroprusside. Data are mean $+\mathrm{SE}$ for three subjects. 
tectable effect on the increases in blood pressure during static handgrip (11). However, increased stimulation of only carotid baroreceptors, by reflexively lowering central aortic pressure, might have decreased the stimulation of aortic baroreceptors and thus masked a potential reflex effect of carotid baroreceptor stimulation (32). In contrast, our pharmacologic manipulations in systemic arterial pressure should have had parallel, not opposing, effects on carotid sinus and aortic arch baroreceptors.

A characteristic feature of the MSNA response to static handgrip is a pronounced latency of almost $60 \mathrm{~s}$ from the onset of exercise to the onset of sympathetic activation $(20,24)$. Previous studies have suggested that this latency is directly related to the slow accumulation of ischemic metabolites in the vicinity of the metaboreceptor afferent endings $(20,24)$. Our findings indicate that this latency also is related in part to baroreflex buffering.

Our neurophysiologic data in humans confirm and extend previous conclusions based upon hemodynamic data in experimental animals $(13,14)$. In conscious dogs, acute, but not chronic, sinoaortic denervation augmented the increases in vascular resistance and blood pressure during submaximal dynamic exercise (14-16). In anesthetized, paralyzed dogs with aortic baroreceptor denervation, carotid sinus hypertension attenuated and carotid sinus hypotension augmented the reflex increases in vascular resistance and blood pressure elicited by electrically induced (i.e., not contraction-induced) stimulation of sciatic nerve afferents, suggesting an interaction between these inhibitory and excitatory reflexes (13).

Similarly, our findings in humans indicate that during static handgrip baroreflex inhibition offsets more than $75 \%$ of the excitatory neural drive caused by the stimulation of metaboreceptor muscle afferents. The magnitude of this inhibition is considerably larger than that expected from a simple summation of excitatory and inhibitory reflexes: the sympathetic excitation caused by handgrip during infusion of nitroprusside was more than two times greater than the simple algebraic sum of the excitation caused by handgrip alone and that caused by infusion of nitroprusside alone, supporting the concept of a reflex interaction. This interaction between muscle metaboreceptor and sinoaortic baroreceptor reflexes in the regulation of MSNA stands in contrast to the recently reported lack of such interaction between the muscle metaboreceptor reflex and cardiopulmonary reflexes $(29-31,33)$.

Although these experiments in humans do not elucidate the underlying mechanism of this reflex interaction, data from experimental animals suggest at least two different possibilities. First, the central projections of the muscle metaboreceptor and of the sinoaortic baroreceptor afferents may converge on some of the same supraspinal neuronal sympathetic pools, providing the anatomic substrate for altered central processing of these afferent inputs $(34,35)$. A second possibility is that the muscle metaboreceptor reflex activates efferent sympathetic traffic not only to skeletal muscle but also to the sinoaortic baroreceptors, causing baroreceptor sensitization (i.e., augmented baroreceptor discharge at a given level of arterial pressure). In the ex vivo rat aortic arch-aortic nerve preparation, single fiber baroreceptor discharge is augmented by norepinephrine (36). In dogs, carotid baroreceptor discharge is augmented by electrical or by reflex stimulation of the cervical sympathetic nerves that innervate the carotid sinus $(37,38)$.
Many previous studies have indicated that exercise causes a rather selective attenuation of the heart rate component (as opposed to the peripheral sympathetic component) of the sinoaortic baroreflex $(9-11,14,17)$. During exercise, brief stimulation of carotid baroreceptors evokes a greatly attenuated reflex decrease in heart rate but normal or possibly augmented reflex decreases in sympathetic outflow, vascular resistance, and arterial pressure. During static handgrip, however, our sustained perturbations in sinoaortic baroreceptor stimulation markedly affected heart rate as well as MSNA.

This unexpected finding may have important implications regarding the interplay between the different mechanisms that regulate heart rate and MSNA during static exercise. Several previous publications have suggested that during static handgrip heart rate, in contrast to MSNA, is governed mainly by central command rather than by metaboreceptor muscle afferents $(20,27)$. This formulation is based in part upon two observations: $(a)$ heart rate increases rapidly with the initiation of motor effort and shows very little additional increase during the second minute of sustained handgrip (when MSNA is increasing progressively); and (b) this exercise-induced tachycardia is not maintained during posthandgrip forearm circulatory arrest, a maneuver that maintains the stimulation of muscle metaboreceptor afferents but eliminates central command (20). In contrast, when the experimental protocol was performed during pharmacologic reduction in baroreceptor activation (infusion of nitroprusside) heart rate, like MSNA, showed a marked and progressive increase throughout the second minute of sustained handgrip; and this augmented increase in heart rate, like the augmented increase in MSNA, was partially maintained during posthandgrip forearm circulatory arrest. These findings suggest that the sinoaortic baroreflex normally may mask an excitatory effect of muscle metaboreflex stimulation on sinus node function.

In conclusion, this study indicates that in healthy humans the sinoaortic baroreflex is much more effective than previously thought in buffering the reflex sympathetic activation caused by static muscle contraction. These experimental findings suggest that in the clinical setting impaired baroreflex function might lead to exaggerated reflex increases in sympathetic outflow and arterial pressure during exercise in patients with hypertension, heart failure, or advancing age.

\section{Acknowledgments}

The authors are indebted to Dr. Jere H. Mitchell for his continued support and critical review of this work, to Ms. Patricia Powell for expert secretarial assistance, and to Eric Clough for superb research assistance.

This research was supported by grants from the Foundation Suisse de Bourses en Médecine et Biologie, and the Policlinique Médicale Universitaire, Lausanne, Switzerland (U. Scherrer) National Heart, Lung, and Blood Institute Program Project grant HL-06296 (R. Victor) training grant HL-07360 (L. Bertocci); Clinical Investigator Award HL-01886 (R. Victor); grants from the American Heart AssociationTexas Affiliate (S. Pryor, R. Victor); and the Lawson and Rogers Lacy Research Fund in Cardiovascular Diseases, University of Texas Southwestern Medical Center. Dr. Victor is an Established Investigator of the American Heart Association.

\section{References}

1. Lind, A. R., R. W. Humphreys, B. M. Kennelly, and K. W. Donald. 1964. The circulatory effects of sustained voluntary muscle contraction. Clin. Sci. (Lond.). 27:229-244. 
2. Martin, C. E., J. A. Shaver, D. F. Leon, M. E. Thompson, P. S. Reddy, and J. J. Leonard. 1974. Autonomic mechanisms in hemodynamic responses to isometric exercise. J. Clin. Invest. 54:104-115.

3. Krogh, A., and J. Lindhard. 1913. The regulation of respiration and circulation during the initial stages of muscular work. J. Physiol. (Lond.). 47:112-136.

4. Goodwin, G. M., D. I. McCloskey, and J. H. Mitchell. 1972. Cardiovascular and respiratory responses to changes in central command during isometric exercise at constant muscle tension. J. Physiol. (Lond.). 226:173-190.

5. Kaufman, M. P., J. C. Longhurst, K. J. Rybicki, J. H. Wallach, and J. H. Mitchell. 1983. Effects of static muscle contraction on impulse activity of group III and IV afferents in cats. J. Appl. Physiol. 55:105-112.

6. Mitchell, J. H., and R. F. Schmidt. 1983. Cardiovascular reflex control by afferent fibers from skeletal muscle receptors. In Handbook of Physiology. Volume III, Part 2. J. T. Shepherd, and F. M. Abboud, editors. American Physiological Society, Bethesda, MD. 623-658.

7. Victor, R. G., D. M. Rotto, S. L. Pryor, and M. P. Kaufman. 1988. Stimulation of renal sympathetic activity by static contraction: evidence for mechanoreceptor-induced reflexes from skeletal muscle. Circ. Res. 64:592-599.

8. Bevegård, B. S., and J. T. Shepherd. 1966. Circulatory effects of stimulating the carotid arterial stretch receptors in man at rest and during exercise. J. Clin. Invest. 45:132-142.

9. Bristow, J. D., E. B. Brown, Jr., D. J. C. Cunningham, M. G. Howson, E. S. Petersen, T. G. Pickering, and P. Sleight. 1971. Effects of bicycling on the baroreflex regulation of pulse interval. Circ. Res. 28:582-592.

10. Ludbrook, J., I. B. Faris, J. Iannos, G. G. Jamieson, and W. J. Russell. 1978. Lack of effect of isometric handgrip exercise on the responses of the carotid sinus baroreceptor reflex in man. Clin. Sci. Mol. Med. 55:189-194.

11. Mancia, G., G. A. Ferrari, L. Gregorini, G. Parati, and G. Pomidossi. 1982. Effects of isometric exercise on the carotid baroreflex in hypertensive subjects. Hypertension (Dallas). 4:245-250.

12. Ebert, T. J. 1986. Baroreflex responsiveness is maintained during isometric exercise in humans. J. Appl. Physiol. 61:797-803.

13. Abboud, F. M., A. L. Mark, and M. D. Thames. 1981. Modulation of the somatic reflex by carotid baroreceptors and by cardiopulmonary afferents in animals and humans. Circ. Res. Suppl. 48:I-131I-137.

14. Walgenbach, S. C., and D. E. Donald. 1983. Inhibition by carotid baroreflex of exercise-induced increases in arterial pressure. Circ. Res. 52:253-262.

15. McRitchie, R. J., S. F. Vatner, D. Boettcher, D. Heyndrickx, T. A. Patrick, and E. Braunwald. 1976. Role of arterial baroreceptors in mediating cardiovascular response to exercise. Am. J. Physiol. 230:85-89.

16. Krasney, J. A., M. G. Levitzky, and R. C. Koehler. 1974. Sinoaortic contribution to the adjustment of systemic resistance in exercising dogs. J. Appl. Physiol. 36:679-685.

17. Eckberg, D. L., and B. G. Wallin. 1987. Isometric exercise modifies autonomic baroreflex responses in humans. J. Appl. Physiol. 63:2325-2330.

18. Delius, W., K.-E. Hagbarth, A. Hongell, and B. G. Wallin. 1972. Manoeuvers affecting sympathetic outflow in human nerves. Acta Physiol. Scand. 84:82-94.

19. Vallbo, A. B., K.-E. Hagbarth, H. E. Torebjörk, and B. G. Wallin. 1979. Somato-sensory, proprioceptive, and sympathetic activity in human peripheral nerves. Physiol. Rev. 59:919-957.

20. Mark, A. L., R. G. Victor, C. Nerhed, and B. G. Wallin. 1985.
Microneurographic studies of the mechanisms of sympathetic nerve responses to static exercise in humans. Circ. Res. 57:461-469.

21. Moon, R. B., and J. H. Richards. 1973. Determination of intracellular $\mathrm{pH}$ by $31 \mathrm{P}$ nuclear magnetic resonance. J. Biol. Chem. 284:7268-7276.

22. Wiener, D. H., J. Maris, B. Chance, and J. R. Wilson. 1986. Detection of skeletal muscle hypoperfusion during exercise using phosphorus-31 nuclear magnetic resonance spectroscopy. J. Am. Coll. Cardiol. 7:793-799.

23. Hands, L. H., P. J. Bore, G. Galloway, P. J. Morris, and G. K. Radda. 1986. Muscle metabolism in patients with peripheral vascular disease investigated by $31 \mathrm{P}$ nuclear magnetic resonance spectroscopy. Clin. Sci. (Lond.). 71:283-290.

24. Victor, R. G., L. A. Bertocci, S. L. Pryor, and R. L. Nunnally. 1988. Sympathetic nerve discharge is coupled to muscle cell $\mathrm{pH}$ during exercise in humans. J. Clin. Invest. 82:1301-1305.

25. Sinoway, L., S. Prophet, I. Gorman, T. Mosher, J. Shenberger, M. Dolecki, R. Briggs, and R. Zelis. 1989. Muscle acidosis during static exercise is associated with calf vasoconstriction. J. Appl. Physiol. 66:429-36.

26. Rotto, D. M., and M. P. Kaufman. 1988. Effects of metabolic products of muscle contraction on discharge of group III and IV muscle afferents. J. Appl. Physiol. 64:2306-2313.

27. Shepherd, J. T., C. G. Blomqvist, A. R. Lind, J. H. Mitchell, and B. Saltin. 1981. Static (isometric) exercise: retrospection and introspection. Circ. Res. Suppl. 48:I-179-I-188.

28. Victor, R. G., S. L. Pryor, N. H. Secher, and J. H. Mitchell. 1989. Effects of partial neuromuscular blockade on sympathetic nerve responses to static exercise in humans. Circ. Res. 65:468-476.

29. Scherrer, U., S. Floistrup Vissing, and R. G. Victor. 1988. Effects of lower-body negative pressure on sympathetic responses to static exercise in humans. Microneurographic evidence against cardiac baroreflex modulation of the exercise pressor reflex. Circulation. 78:49-59.

30. Seals, D. R. 1988. Cardiopulmonary baroreflexes do not modulate exercise-induced sympathoexcitation. J. Appl. Physiol. 64:21972203.

31. Sanders, J. S., and D. W. Ferguson. 1988. Cardiopulmonary baroreflexes fail to modulate sympathetic responses during isometric exercise in humans: direct evidence from microneurographic studies. J. Am. Coll. Cardiol. 12:1241-1251.

32. Sanders, J. S., A. L. Mark, and D. W. Ferguson. 1989. Importance of aortic baroreflex in regulation of sympathetic responses during hypotension: evidence from direct sympathetic nerve recordings in humans. Circulation. 79:83-92.

33. Seals, D. R., N. O. Suwarno, and J. A. Dempsey. 1990. Influence of lung volume on sympathetic nerve discharge in normal humans. Circ. Res. 67:130-141.

34. Abboud, F. M., and M. D. Thames. 1983. Interaction of cardiovascular reflexes in circulatory control. In Handbook of Physiology. Volume III, Part 2. J. T. Shepherd, and F. M. Abboud, editors. American Physiological Society, Bethesda, MD. 675-753.

35. Kalia, M., S. S. Mei, and F. F. Kao. 1981. Central projections from ergoreceptors ( $C$ fibers) in muscle involved in cardiopulmonary responses to static exercise. Circ. Res. Suppl. 48:I-48-I-62.

36. Munch, P. A., P. N. Thorén, and A. M. Brown. 1987. Dual effects of norepinephrine and mechanisms of baroreceptor stimulation. Circ. Res. 61:409-419.

37. Bolter, C. P., and J. R. Ledseme. 1976. Effect of cervical sympathetic nerve stimulation on canine carotid sinus reflex. Am. J. Physiol. 230:1026-1030.

38. Felder, R. B., C. M. Heesch, and M. D. Thames. 1983. Reflex modulation of carotid sinus baroreceptor activity in the dog. Am. J. Physiol. 244:H437-H443. 